\title{
Errata: Exclusion Process and Droplet Shape ${ }^{1}$
}

\author{
J.-P. Marchand ${ }^{2}$ and Ph. A. Martin ${ }^{2}$
}

Received August 28, 1987

In the Introduction it was incorrectly stated that the asymptotic measure $v_{\infty}(\eta)$ of the exclusion process with initial measure $v_{0}(\eta)$ concentrated on the configuration

$$
\eta_{0}(k)= \begin{cases}1 & k \leqslant 0 \\ 0 & k>0\end{cases}
$$

is a product measure. This error entails the following corrections:

Page 492, lines 6-14: The system behaves differently for $r=p / q$ larger or smaller than one. If $r \geqslant 1$, the system starting from $\eta_{0}$ approaches the product measure with density $1 / 2$. If $r<1 ; v_{\infty}(\eta)$ is obtained by conditioning the product measure

$$
\prod_{l: \eta(l)=1} \rho(l) \prod_{l: \eta(l)=0}[1-\rho(l)]
$$

with

$$
\rho(l)=r^{l}\left(1+r^{l}\right)^{-1}
$$

on the set $X_{0}$ of configurations that can be reached from $\eta_{0}$.

Page 496, lines 5-6: Let $\Omega_{0}$ be the set of configurations $\omega \in \Omega$ that can be reached from $\omega_{0}$, and $\mu_{\infty}$ the equilibrium measure on $\Omega_{0}$ (corresponding to $v_{\infty}$ on $X_{0}$ ).

Moreover, the last term of Eq. (2.3) on p. 497 should read $\frac{1}{2} c \prod_{1}^{\infty}\left(1+r^{l}\right)^{-2}$, and this implies that formula (2.7) must be replaced by

$$
c=2 \pi(1, \infty) \prod_{1}^{\infty}\left(1+r^{l}\right)^{2}
$$

\footnotetext{
${ }^{1}$ This paper appeared in J. Stat. Phys. 44:491 (1986).

${ }^{2}$ University of Denver, Colorado, and Ecole Polytechnique Fédérale de Lausanne, Switzerland.
} 
Comments. The measure $v_{\infty}(\eta)$ differs from $(1.1)$ by the multiplicative constant $c$ introduced in Lemma 2.1, with the above value (2.7), and $\rho(l)$ is not equal to $v_{\infty}(\eta: \eta(l)=1)$. The heuristic derivation of $(3.3)$ presented on p. 501 is incorrect, since it uses $\rho(l)$ instead of the correct expression for $v_{\infty}(\eta: \eta(l)=1)$, which is much more involved. But the formula (3.3) itself and the remainder of Section 3 are correct, since they are a consequence of the correct relation (Proposition 2.8)

$$
b_{k}(n)=r^{k n} \pi(k, \infty) \pi^{-1}(1, n)
$$

[with $\pi(1,0)=1$ ], which may be rederived more directly as follows:

Considering Fig. 1, one finds that the number of boundaries enclosing an area $l$ are $q_{l}^{(k-1)}$ and $q_{l}^{(n)}$ for regions II and III, respectively. Hence the total weight of all boundaries satisfying $B_{k}(\omega)=n$ is [cf. Lemma 2.1, formula (2.6)]

$$
\begin{aligned}
b_{k}(n) & =\pi(1, \infty) r^{k n}\left(\sum_{l=0}^{\infty} q_{l}^{(k-1)} r^{l}\right)\left(\sum_{l=0}^{\infty} q_{l}^{(n)} r^{l}\right) \\
& =\pi(1, \infty) r^{k n} \hat{q}^{(k-1)}(r) \hat{q}^{(n)}(r)
\end{aligned}
$$

from which the result follows by (2.9).

As a final remark, we emphasize that the isomorphism between the processes $v_{t}(\eta)$ on $X_{0}$ and $\mu_{t}(\eta)$ on $\Omega_{0}$ is due to $\mathrm{H}$. Rost ${ }^{(2)}$ for $p=1$ and appears in Chapter VIII of Ref. 1 for general $p$.

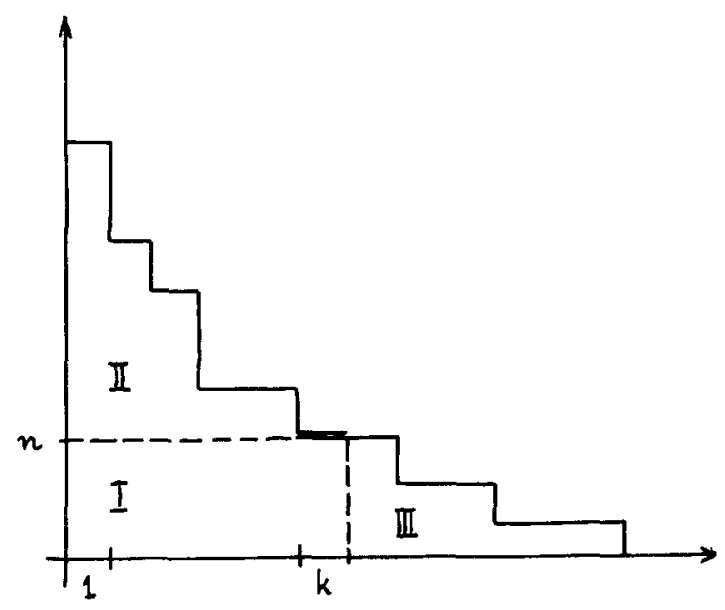

Fig. 1. 


\section{ACKNOWLEDGMENT}

We thank Prof. T. Liggett for informing us about our error.

\section{REFERENCES}

1. T. M. Liggett, Interacting Particle System, (Springer-Verlag, 1985).

2. H. Rost, Z. Wahrsch. Verw. Gebiete 58:41 (1981). 